4. Wang J, Arber D, Znati K, El Fatemi H, Harmouch T, Zaki Z, et al. Large solitary fibrous tumor of the kidney. Am J Surg Pathol. 2001;25:1194-9.

5. Xambre L, Lages R, Cerqueira M, Silva V, Prisco R, Santos R, et al. Tumor fibroso solitario. Dos casos adicionales con implicancias urológicas. Actas Urol Esp. 2003;27: 832-8.

6. Magro G, Cavallaro V, Torrisi A, Lopes M, Dell'Albani M, Lanzafame S. Intrarenal solitary fibrous tumor of the kidney report of a case with emphasis on the differential diagnosis in the wide spectrum of monomorphous spindle cell tumors of the kidney. Pathol Res Pract. 2002;198:37-43.

7. Yasaki T, Satoh S, Iizumi T, Umeda T, Yamaguchi Y. Solitary fibrous tumor of renal pelvis. Int J Urol. 2001;8:504-8.

8. Llarena R, Eizaguirre B, Zarzai B, Lecumberri Castanos D, et al. Bilateral renal solitary fibrous tumor. Arch Esp Urol. 2003;56: $835-40$.
9. Graadt van Roggen JF, Hogendoorn PCW. Solitary fibrous tumour: The emerging clinicopathologic spectrum of an entity and its differential diagnosis. Curr Diagn Pathol. 2004;10:229-35.

10. Torabi A, Lele SM. Lack of a common or characteristic cytogenetic anomaly in solitary fibrous tumor. Cancer Genet Cytogenet. 2008;181:60-4.

L. Taxa ${ }^{a}$, L. Huanca ${ }^{a, *}$, L. Meza ${ }^{b}$ y M. Pow Sang ${ }^{b}$

${ }^{a}$ Departamento de Patología, Instituto Nacional

de Enfermedades Neoplásicas, Lima, Perú

${ }^{\mathrm{b}}$ Departamento de Cirugía Urológica, Instituto Nacional

de Enfermedades Neoplásicas, Lima, Perú

*Autor para correspondencia.

Correo electrónico: Lulita1411@gmail.com (L. Huanca).

\title{
Extrusión de prótesis testicular: presentación de un caso y revisión de la literatura médica
}

\section{Extrusion of a testicular prosthesis: Presentation of a clinical note and review of the literature}

\section{Sr. Director:}

Presentamos el caso de un paciente varón de 23 años de edad que acudió al Servicio de Urgencias de nuestro hospital por presentar una tumoración en el hemiescroto izquierdo, acompañada de intenso dolor y de dos días de evolución. Entre sus antecedentes personales refería haber sufrido un traumatismo testicular izquierdo a los 9 años de edad, siendo intervenido 2 meses después de este.

Dos días antes de acudir a nuestro hospital consultó con su médico de atención primaria por presentar una tumoración en el hemiescroto izquierdo de aproximadamente $0,5 \mathrm{~cm}$ de diámetro y que se acompañaba de signos inflamatorios cutáneos. Fue diagnosticado de quiste sebáceo escrotal complicado y tratado con antibióticos y antiinflamatorios. Veinticuatro horas después de iniciar el tratamiento médico observó la presencia de una escara negruzca en el centro de la tumoración que aumentó de tamaño en el plazo de varias horas y que, al desprenderse, dejó visible el material protésico.

En la exploración física, el paciente se encontraba apirético y normotenso. Se apreció la extrusión parcial de una prótesis testicular en la región superoexterna del hemiescroto izquierdo, el cual no presentaba signos inflamatorios ni hemorragia (fig. 1). Sorprendentemente, el paciente desconocía que en la intervención quirúrgica realizada en la infancia le practicaron orquiectomía izquierda e implante de prótesis testicular de silicona. El resto de la exploración era normal.

Bajo anestesia local se procedió a la extracción completa de la prótesis testicular del escroto, al lavado de la cavidad con solución de clorhexidina al 0,1\%, al refrescamiento y a la sutura de los bordes cutáneos. Durante la intervención se apreció la presencia de una pseudocápsula fibrosa y de superficie lisa alrededor del lugar que ocupaba la prótesis. Se revisó la herida a los 7 días, encontrándose en perfectas condiciones. A los 2 meses el paciente se encontraba asintomático. El paciente rechazó la colocación de una nueva prótesis.

La ausencia de un testículo puede suponer para el paciente una experiencia traumática desde el punto de vista psicológico, con sensación de inferioridad, alteraciones en la función sexual y efectos sobre el desarrollo psicosocial. Es más probable que esto ocurra en pacientes que han perdido un testículo que en aquellos que han nacido sin él.

La relativa facilidad de colocación de la prótesis testicular, su similitud con un testículo normal y el bajo grado de complicaciones permiten reconstruir un escroto aparentemente normal en aquellos pacientes que han presentado la pérdida de un testículo o han nacido sin él ${ }^{1}$.

\section{Indicaciones para la implantación de prótesis testicular}

Las causas más frecuentes para la indicación de la colocación de una prótesis son la agenesia o la atrofia testicular. En pacientes con criptorquidia se puede encontrar atrofia o agenesia testicular hasta en un $10 \%$ de los casos. Las causas de indicación de orquiectomía y la colocación de una prótesis testicular según una encuesta realizada a los urólogos de la sección occidental de la AUA en $1986^{2}$ son el testículo atrófico o no descendido (35\%), la torsión testicular (23\%), el tumor 


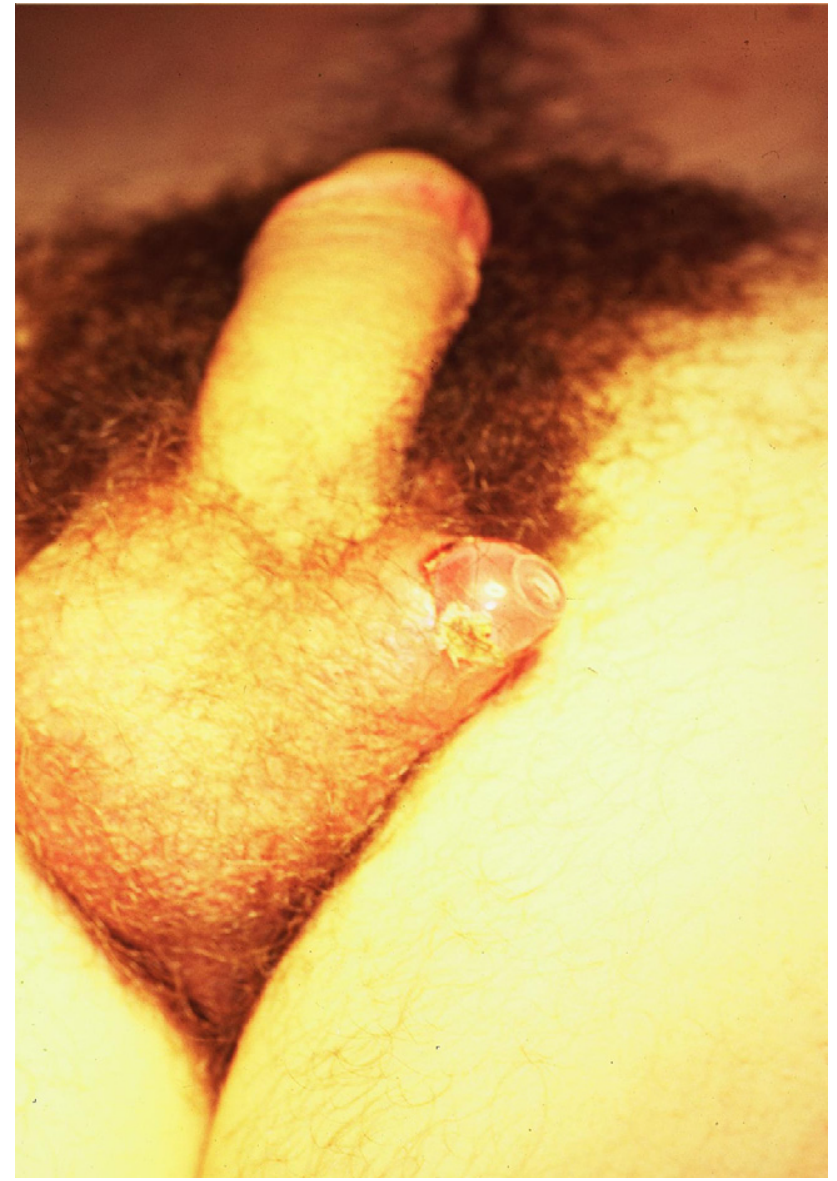

Figura 1 - Prótesis testicular desplazada y fistulizada con la piel escrotal.

testicular (17\%) y el cáncer de próstata metastático (16\%). Actualmente, la última indicación mencionada casi ha desaparecido de la práctica urológica habitual.

\section{Tipos de prótesis}

Hoy en día se emplean dos tipos de prótesis: las de silicona y las rellenas de gel de silicona o elastómero de suero salino fisiológico. Debido a las complicaciones de los implantes mamarios de silicona, actualmente en Estados Unidos solamente está aprobada la prótesis de suero salino ${ }^{3}$. Sin embargo, no existen estudios que demuestren poder carcinógenico ni mayor grado de complicaciones de las prótesis de silicona ${ }^{4}$. Por este motivo en Europa se siguen usando las prótesis de silicona. Estas vienen en tres tamaños: pequeño, mediano y grande, y en muchos casos los tamaños mencionados no se ajustan a las características anatómicas del paciente.

Existe cierta controversia en cuanto al momento más idóneo para el implante de una prótesis, sobre todo en los niños. Se debe plantear colocársela lo más joven posible para evitarle traumas durante el crecimiento. Una vez pasada la pubertad se considerará plantear el cambio de esta por una que vaya en relación con el tamaño testicular del momento. Si el paciente se encuentra satisfecho con el tamaño de su primera prótesis, debe evitarse una nueva intervención quirúrgica.

\section{Técnicas}

1. Colocación en una bolsa subcuticular Descrita por Abbassian en $1972^{2,5}$. Se indica en aquellos casos donde se encuentra el hemiescroto vacío muy atrófico o con tejido cicatricial. Se realiza una incisión de $2-3,5 \mathrm{~cm}$ en el hemiescroto contralateral sin sobrepasar el rafe medio y se obtiene a través de esta una bolsa subcuticular. Después de la inserción de la prótesis, se cierra el rafe de manera longitudinal con una sutura absorbible. La principal complicación de este tipo de intervención es el alto porcentaje de extrusión de la prótesis.

2. Incisión transescrotal, orquiectomía y preservación del conducto deferente

Descrita por Solomon ${ }^{2}$. Se realiza en el mismo tiempo quirúrgico de la orquiectomía, pero se preservan el gubernaculum, el epidídimo y las estructuras del cordón, estando contraindicado en casos de tumores testiculares. Se realiza una incisión transescrotal hasta la abertura de la túnica albugínea. Se colocan puntos de fijación en la túnica vaginalis. Se procede a la orquiectomía y a la sutura de la prótesis al gubernaculum y al epidídimo. Se cierra la incisión por planos.

3. Abordaje inguinal o inguinoescrotal Descrito por Lattimer en $1973^{2,6}$. Es la más empleada por la mayoría de urólogos en la actualidad. Se realiza una incisión inguinal baja o escrotal alta, que debe quedar lejos de la zona de reposo de la prótesis para evitar la extrusión. Se obtiene una bolsa del escroto con dos dedos. Con una pinza de Allis se plica la base del escroto y se invierte usando como guía el dedo índice. Mediante esta maniobra se puede colocar de manera eficaz la sutura de fijación. Se aplica la sutura mencionada en el escroto estando seguros de incluir el dartos pero sin perforar la piel para evitar riesgo de infecciones. Se pasa esta sutura a través del anillo de fijación de la prótesis. Se coloca la prótesis en la base del escroto. Una vez comprobada la ausencia de perforación en la piel se procede a cerrar el cuello del escroto con suturas reabsorbibles en varias capas. En aquellos casos donde se encuentre un hemiescroto retraído, se puede utilizar una torunda, un balón de sonda de Foley o un dilatador tipo Hegar para crear mayor espacio en el hemiescroto correspondiente ${ }^{7}$.

\section{Complicaciones}

El estudio con mayor número de casos (2.500) sobre las complicaciones de los implantes de las prótesis testiculares fue llevado a cabo por Marshall en 1986 a través de un cuestionario a urólogos que realizaban este procedimiento en Estados Unidos ${ }^{1}$. La dehiscencia de la herida quirúrgica con extrusión de la prótesis es la complicación más común (3-8\%) y se produce en el período postoperatorio tardío. Marshall encontró la mayor proporción de esta complicación en aquellos casos que se asociaban a orquiectomía por orquiepididemitis, sobre todo si se había utilizado una incisión escrotal para la colocación de la prótesis. Asimismo, se 
encontró mayor riesgo de complicaciones en general en aquellos pacientes sometidos a cirugía escrotal previa y también si existía un lapso muy largo entre la orquiectomía y la colocación de la prótesis. Otra complicación tardía es la falta de distensibilidad del escroto con el desplazamiento de la prótesis a una posición más alta (3-5\%). Entre las complicaciones inmediatas se encuentran el dolor (1-3\%), el hematoma (0,3-3\%) y la infección de la herida (0,6-2\%). En nuestro caso, la extrusión de la prótesis probablemente se produjo por un fallo de su colocación en el punto de mayor declive en el hemiescroto correspondiente, bien debido a la colocación en una zona alta o bien debido a una retracción escrotal con ascenso de la prótesis. En cualquiera de los casos, esta situación favorece la aparición de un decúbito escrotal y/o a que una infección cutánea facilite la extrusión. Se ha publicado algún caso de rotura de la prótesis de silicona (muy infrecuente sin daño de esta durante la cirugía) $)^{4}$ e incluso algún caso de rotura espontánea de una prótesis 11 años después de su colocación ${ }^{8}$.

La colocación de prótesis testiculares ha disminuido el impacto psicológico en aquellos pacientes con ausencia o pérdida de un testículo. La implantación es más simple cuando es llevada a cabo en el momento de la orquiectomía. En aquellos pacientes en los que se restaura en otro acto quirúrgico existe una mayor tasa de complicaciones, siendo la más común de ellas la extrusión de la prótesis testicular. Hasta el momento no se ha encontrado asociación entre la aplicación de prótesis de silicona y efectos carcinogénicos o inmunogénicos. Es fundamental una cuidadosa técnica quirúrgica para minimizar el riesgo de extrusión.

\section{B I B L I O G R A F Í A}

1. Marshall S. Potential problems with testicular prostheses. Urology. 1986;28:388-90.

2. Beer M, Kay R. Testicular prostheses. Urol Clin North Am. 1989;16:133-7.

3. Turek PJ, Master VA, Testicular Prosthesis Study Group. Safety and effectiveness of a new saline filled testicular prosthesis. J Urol. 2004;172:1427-30.

4. Bodiwala D, Summerton DJ, Terry TR. Testicular prostheses: Development and modern usage. Ann R Coll Surg Engl. 2007;89:349-53.

5. Abbassian A. A new surgical technique for testicular implantation. J Urol. 1972;107:618.

6. Lattimer JK, Vakili BF, Smith AM, Morishima A. A natural feeling testicular prosthesis. J Urol. 1973;110:81-3.

7. Elkabir JJ, Smith GL, Dinneen MD. Testicular prosthesis placement: A new technique. BJU Int. 1999;84:867-8.

8. Twidwell J. Ruptured testicular prosthesis. J Urol. 1994;152: 167-8.

J. Reinoso Elbers ${ }^{\text {a,* }}$, J.M. Alonso Dorrego ${ }^{a}$, M. Pérez-Utrilla ${ }^{\mathrm{b}}$ y M. Girón De Francisco ${ }^{\mathrm{a}}$

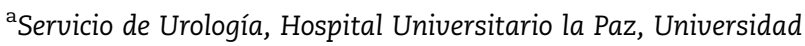
Autónoma de Madrid, Madrid, España

${ }^{\text {b }}$ Servicio de Urología, Hospital General de Ciudad Real, Ciudad Real, España

*Autor para correspondencia.

Correo electrónico: javier.reinoso@gmail.com

(J. Reinoso Elbers). 